\title{
LOCUST IMAGE SEGMENTATION USING PULSE-COUPLED NEURAL NETWORK
}

\author{
Xuemei Xiong, Yiming Wang \\ College of Information and Electrical Engineering, China Agricultural University, 100083 \\ Beijing, China
}

Abstract: The main objective of this study was to evaluate the feasibility of identifying locusts by the image segmentation method based on pulse-coupled neural network (PCNN). Segmentation results by simplified PCNN and traditional morphology open operation method were given. The simulation results demonstrated that the PCNN was powerful enough to perform the detecting.

Keywords: Pulse-coupled neural network, Image segmentation, Locusts detection

\section{PCNN MODEL}

In this work, a PCNN with simplified parameters was applied to locust image segmentation. The area recognition rate (ARR) was used to measure the performance of this method.

\section{EXPERIMENTS ON LOCUST IMAGES}

The performance of PCNN on locust images is shown in Table 1. The best quality of image segmentation for tiny objects (Fig. 1) is obtained by a relatively larger linking strength $\beta$. The time response is different depending on the coupling strength between neighboring neurons and strong coupling neurons have a tendency to fire together for similar stimulus. 
Table 1. Performance of PCNN on locust images

\begin{tabular}{clcc}
\hline Images in & \multicolumn{1}{c}{ Parameters } & Area Recognition Rate & Average Processing Time \\
\hline \multirow{2}{*}{ Fig. 1} & $\Delta T=0.02, V_{T}=100$, & \\
& $\beta=0.8, \alpha=0.2$ & $92 \%$ & $1 \mathrm{~s}$ \\
& $\Delta T=0.02, V_{T}=100$, & & \\
Fig. 2 & $\beta=0.8, \alpha=0.2$ & $81 \%$ & $1 \mathrm{~s}$ \\
\hline
\end{tabular}

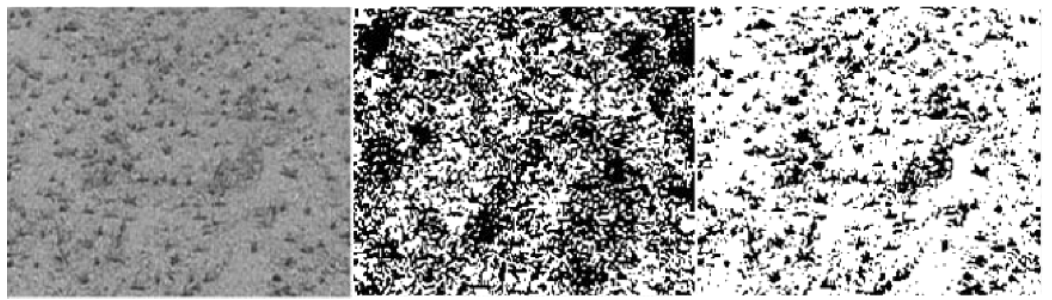

Fig. 1. Locusts from a long distance (left, converted as a gray-scale image) and the segmentation results (middle: result of open operation; right: result of PCNN at $n=2$ )

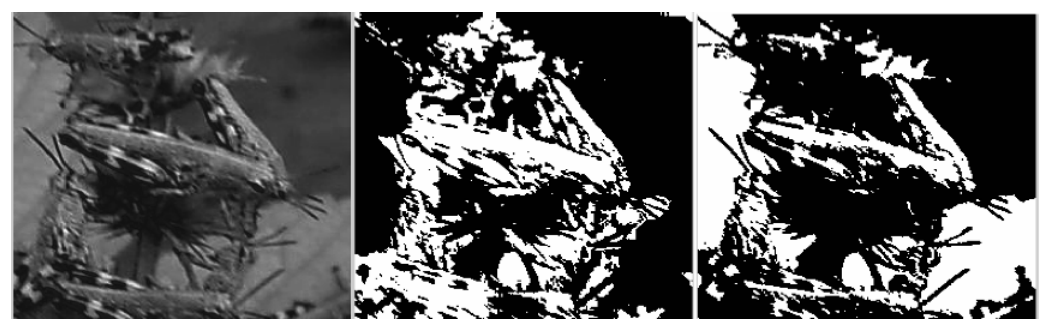

Fig. 2. Locusts from a short distance (left, converted as a gray-scale image) and the segmentation results (middle: result of open operation; right: result of PCNN at $n=2$ )

\section{CONCLUSION}

We applied the simplified PCNN algorithm to locust image segmentation. The performance of PCNN is compared with the traditional morphology open operation method. Experimental results of PCNN in terms of visual effects are better than the open operation of mathematic morphology. 